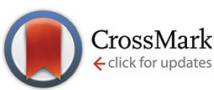

Cite this: Org. Biomol. Chem., 2015, 13,10244

\title{
Bioorthogonal phase-directed copper-catalyzed azide-alkyne cycloaddition (PDCuAAC) coupling of selectively cross-linked superoxide dismutase dimers produces a fully active bis-dimer $\dagger$
}

\begin{abstract}
Erika M. J. Siren, Serena Singh and Ronald Kluger*
Superoxide dismutase (SOD) is a $32 \mathrm{kDa}$ dimeric enzyme that actively removes a toxic oxygen species within red cells. The acellular protein itself does not survive circulation as it is filtered through the kidney. Conjugating the protein to another SOD should increase the size of the dual protein above the threshold for filtration by the kidney, making the material a potential therapeutic in circulation. Site-selective chemical cross-linking of SOD introduces a bioorthogonal azide group on the cross-link so that two SODs react efficiently with a bis-alkyne through phase-directed copper-catalyzed azide-alkyne cycloaddition (PDCUAAC). The modification has a negligible effect on the catalytic activity of the constituent proteins. Consistent with the retained activity, circular dichroism (CD) spectroscopy indicates that the secondary structures of the proteins are similar to that of the native protein.
\end{abstract}

Received 12th August 2015, Accepted 18th August 2015 DOI: $10.1039 / \mathrm{c5}$ ob01702e www.rsc.org/obc

\section{Introduction}

While $[\mathrm{Cu}, \mathrm{Zn}]$ superoxide dismutase (SOD) possesses great potential as a therapeutic detoxification agent, unfavourable properties in circulation have limited its clinical implementation. ${ }^{1,2}$ The small, water-soluble dimer is excreted as it passes through the kidney in renal circulation. ${ }^{3,4}$ Previous attempts to delay excretion focused on covalent addition of polyethylene glycol to thiols of cysteine side chains. ${ }^{5,6}$ Saifer and coworkers showed that modification of SOD with $35 \mathrm{kDa}$ PEG extends the half-life of SOD in circulation from less than 10 minutes to over $24 \mathrm{~h}^{7}$ However, the resulting modified protein consists of mixtures that are difficult to characterize and that may elicit an inflammatory response. ${ }^{8,9}$ In order to increase the viability of $[\mathrm{Cu}, \mathrm{Zn}]$ SOD as a therapeutic agent, we adopted a bioorthogonal strategy to produce a size-doubled functional protein (to maintain it in circulation) based on modern chemical methods of bioorthogonal copper-catalyzed cycloaddition reactions that combine azides and alkynes. ${ }^{10-13}$

Our approach involves chemical introduction of specific functional sites for phase-directed copper-catalyzed azidealkyne cycloaddition (PDCuAAC) to effect site-directed proteinprotein coupling. ${ }^{14}$ This approach utilizes a bis-alkyne that has very limited water-solubility undergoing CuAAC reaction with

Davenport Laboratories, Department of Chemistry, University of Toronto, Toronto, Ontario, Canada M5S 3H6.E-mail: rkluger@chem.utoronto.ca

$\dagger$ Electronic supplementary information (ESI) available: Mass spectrometry data. See DOI: 10.1039/c5ob01702e the protein-coupled azide at the interface between the aqueous and organic phases. Once the initial CuAAC reaction occurs, the remaining alkyne is brought into the aqueous phase by the attachment of the other end to the protein. In a common phase, an azide and the remaining alkyne react much more rapidly, assuring the success of protein-protein coupling. We prepared a specifically cross-linked SOD dimer by functional group-directed sterically selective acylation of the protein with a bifunctional azide. This is coupled to another azide-modified SOD using an electron-deficient bis-alkyne linker and PDCuAAC. ${ }^{10-13}$ The resulting species has the exact mass expected for a coupled $64 \mathrm{kDa}$ entity, which exceeds the threshold size that is needed for a protein to remain in circulation without interference from renal filtration (30-50 kDa). ${ }^{15}$

\section{Experimental}

\section{General methods}

Unless otherwise noted, commercial reagents were used without further purification. Water was doubly distilled and deionized prior to use. Purified bovine [ $\mathrm{Cu}, \mathrm{Zn}]$ superoxide dismutase (SOD1) was obtained from MP Biomedicals Canada. Protein concentrations were determined using the Bio-Rad protein assay (see below for further details). ${ }^{16}$ UV-vis spectra were recorded on a UV-vis spectrophotometer equipped with a thermostatted cell holder. Cross-linker 1 has been previously described. ${ }^{12}$ Synthesis of bis-alkyne 2 was performed according to literature procedures. ${ }^{13}$ High-resolution mass spectrometry 
was performed at the Advanced Instrumentation for Molecular Structure (AIMS) Laboratory, Department of Chemistry at the University of Toronto.

\section{Cross-linking of SOD}

[Cu,Zn] Superoxide dismutase from bovine erythrocytes $(9.6 \times$ $10^{-8} \mathrm{~mol}$ ) was dissolved in $0.6 \mathrm{~mL}$ of $0.01 \mathrm{M}$ sodium borate buffer ( $\mathrm{pH}$ 9.0). 4.5 equivalents of cross-linking reagent 1 dissolved in DMSO $(0.05 \mathrm{M})$ were added to the solution. Stocks of the linker were made so that the final volume of DMSO in the reaction vessel did not exceed $5 \%$. The cross-linking reaction proceeded for 12 hours at room temperature in the dark with constant stirring. Excess reagent was removed though selective acetone precipitation of the enzyme. Six volume equivalents of cold acetone $\left(-20^{\circ} \mathrm{C}\right)$ were added to the aqueous solution. The acetone was added in 3 intervals over 30 minutes to prevent damage to the enzyme. All precipitation procedures were performed on ice. The enzyme was collected by centrifugation at $14.8 \times 1000 \mathrm{~g}$ for $15 \mathrm{~min}$ at $4{ }^{\circ} \mathrm{C}$ to form a light blue pellet. After air drying for 30 minutes to remove residual acetone, the pellet was re-suspended in deionized water and purified via analytical ion exchange using a SynChropak AX300 column $(250 \times 4.6 \mathrm{~mm})$. The collected peak was lyophilized immediately and stored at $0{ }^{\circ} \mathrm{C}$.

\section{Coupling of cross-linked SOD using PDCuAAC}

To make bis-SOD, cross-linked SOD $\left(\mathrm{SOD}-\mathrm{N}_{3}\right)$ was purified and reacted with the bifunctional alkyne linker 2. The coupling reaction was catalysed by $\mathrm{Cu}(\mathrm{I})$, which was prepared in situ using an aliquot of $\mathrm{CuSO}_{4}$ under reducing conditions. The reaction was carried out in $0.02 \mathrm{M}$ phosphate buffer $(\mathrm{pH} 7.4)$ with SOD- $\mathrm{N}_{3}(0.25 \mathrm{mM})$ in the presence of $2 \mathrm{mM}$ bis-alkyne 2 dissolved in DMSO, $3 \mathrm{mM}$ THPTA ligand, and $0.6 \mathrm{mM} \mathrm{CuSO}_{4}$ along with $7 \mathrm{mM}$ ascorbic acid as the reducing agent. The reaction was left in the dark at room temperature for 4 hours, passed through a $0.45 \mu \mathrm{m}$ filter, and exchanged into 0.1 MOPS buffer ( $\mathrm{pH}$ 8.0) via three consecutive rounds of centrifugal filtration (15 minutes, $14 \times 1000 \mathrm{~g}$ ) using a $50 \mathrm{kDa}$ MWCO membrane. Reactions using TBTA followed identical conditions as described above with the exception of the use of a $2: 1$ ligand to $\mathrm{CuSO}_{4}$ ratio. TBTA was dissolved in a $1: 4 t$-butanol : DMSO medium to form a $20 \mathrm{mM}$ stock solution. For trials employing sulfonated bathophenanthroline (batho) ligand, a $0.5 \mathrm{mM}$ SOD- $\mathrm{N}_{3}$ solution (0.02 M phosphate buffer ( $\mathrm{pH}$ 7.4) was thoroughly purged with a steady stream of $\mathrm{N}_{2}$ over 1 hour. The reaction was carried out with $\mathrm{SOD}^{-\mathrm{N}_{3}}(0.25 \mathrm{mM})$ in the presence of $2 \mathrm{mM}$ bis-alkyne 2 dissolved in DMSO, batho ligand $(1.2 \mathrm{mM})$, and $0.6 \mathrm{mM} \mathrm{CuSO}_{4}$ along with $7 \mathrm{mM}$ ascorbic acid. After the addition of reagent, the vessel was purged, sealed, and left for 4 hours. The mixture was then passed through a $0.45 \mu \mathrm{m}$ filter and exchanged into 0.1 MOPS buffer ( $\mathrm{pH}$ 8.0) via three consecutive rounds of centrifugal filtration (15 minutes, $14 \times 1000 \mathrm{~g}$ ) using a $50 \mathrm{kDa}$ MWCO membrane. The yield of the protein coupling step was calculated from the SDS-PAGE gel band intensities, which were quantified using ImageJ software.

\section{Size exclusion chromatography}

Semi-preparative size exclusion chromatography (SEC) (Sephadex G-200, $1000 \times 35 \mathrm{~mm}$ ) under slightly dissociating conditions served in the analysis of SOD- $\mathrm{N}_{3}$ and bis-SOD. The eluent was $37.5 \mathrm{mM}$ Tris, $\mathrm{pH} 7.4$ with $0.5 \mathrm{M}$ magnesium chloride. The effluent was monitored at $254 \mathrm{~nm}$.

\section{Anion exchange chromatography}

Cross-linked SOD was analyzed and purified using an analytical anion exchange SynChropak AX-300 $(250 \times 4.6)$ column with $1.5 \times 10^{-2} \mathrm{M}$ Tris, pH 8.0 buffer (A) and $1.5 \times 10^{-2} \mathrm{M}$ Tris, $1.5 \times 10^{-1} \mathrm{M}$ sodium acetate $\mathrm{pH} 8.0$ buffer (B). The gradient began with $100 \%$ buffer A and ended with $100 \%$ buffer B over 30 minutes. The effluent was monitored at $254 \mathrm{~nm}$.

\section{Bio-Rad protein assay}

The concentration of modified SOD was determined using the Bio-Rad ${ }^{\circledR}$ protein assay. This procedure utilizes a dye that binds to soluble protein containing Tyr, Trp, or Arg residues. ${ }^{16}$ The absorbance of the dye shifts from 465 to $595 \mathrm{~nm}$ upon protein binding. A calibration curve was produced using bovine serum albumin (BSA) in the range of $0.1-1 \mathrm{mg} \mathrm{mL} \mathrm{m}^{-1}$. The amount of protein in solution was then derived by linear regression analysis with concentrations adjusted by a factor of 4.6. The adjustment factor was required to account for the disparity between the amount of reactive Tyr, Trp and Arg residues in BSA and SOD.

\section{SDS-PAGE analysis}

The molecular weights of constituent proteins were estimated using polyacrylamide gel $(10 \%$ Tris- $\mathrm{HCl}$ or $12 \%$ for the native SOD gel) electrophoresis under denaturing conditions as described by Laemmli. ${ }^{17}$ Two-dimensional Tris-HCl polyacrylamide gels were comprised of $10 \%$ (or $12 \%$ for the native SOD gel) separating gel ( $\mathrm{pH} 8.8$ ) and 5\% stacking gel ( $\mathrm{pH}$ 6.8), both with $10 \%$ sodium dodecyl sulfate. Protein samples were treated with 2-mercaptoethanol and sodium dodecyl sulfate. The dimer was further denatured by heating at $95{ }^{\circ} \mathrm{C}$ for $5 \mathrm{~min}$ and centrifuged for 5 minutes at $2 \times 1000 \mathrm{~g}$ before loading onto the gel. Gels were run at $140 \mathrm{~V}$ for approximately 1 hour. Finished gels were stained with Coomassie Brilliant Blue.

\section{Assay of SOD activity}

The method used is as described by Marklund. ${ }^{18}$ Air-equilibrated $56 \mathrm{mM}$ Tris-cacodylic buffer with $1.1 \mathrm{mM}$ DTPA ( $\mathrm{pH}$ 8.2) was prepared by mixing the buffer with air for $24 \mathrm{~h}$. Stock solutions of lyophilized native and modified SOD were prepared using this buffer. The enzymatic reaction was initiated by the addition of pyrogallol (final concentration $4 \mathrm{mM}$ ), which was taken from a $20 \mathrm{mM}$ solution of pyrogallol in $10 \mathrm{mM} \mathrm{HCl}$. The change in absorbance at $420 \mathrm{~nm}$ was followed at $25{ }^{\circ} \mathrm{C}$. The optimal enzyme concentration was determined by establishing a value for one unit of the enzyme, which is defined as the amount that inhibits autoxidation of pyrogallol by $50 \%$. Inhibition was determined according to the 
following equation: $(\%$ inhibition $=(A-B) \times 100 / A) . A$ and $B$ are the initial auto-oxidation rates of pyrogallol in the absence and presence of SOD respectively. ${ }^{19}$ The amount of protein required to inhibit pyrogallol autoxidation by $50 \%$ was defined as 1 unit of enzyme activity. A rate profile was prepared of pyrogallol auto-oxidation in the presence and absence of SOD. To obtain the specific activity (units per $\mathrm{mg}$ ) of SOD, a line was fitted to a set of known concentrations of enzyme against their corresponding \% inhibition values. From this graph, the amount of enzyme (mg) needed for 50\% inhibition was interpolated. ${ }^{20}$ This value was then converted to the overall specific activity of the product (units per $\mathrm{mg}$ ).

\section{Circular dichroism (CD) analysis of modified SOD}

CD analysis was performed with a Jasco J-710 spectropolarimeter using a quartz cuvette $(0.1 \mathrm{~cm}$ path length). Spectra reported are an average of 10 runs. A scan speed of $50 \mathrm{~nm}$ $\min ^{-1}$ and $1.0 \mathrm{~nm}$ bandwidth with a response time of 2 seconds was used. Samples were recorded in $20 \mathrm{mM}$ phosphate buffer $\mathrm{pH} 7.0$ with protein concentrations of $1 \mu \mathrm{M}$.

\section{Results \& discussion}

\section{Cross-linking of SOD}

A bioorthogonal site for site-directed protein-protein coupling was introduced by specifically cross-linking the SOD dimer with the bifunctional azide linker 1 (Scheme 1).

To avoid non-specific acylation, 3,5-dibromosalicylate moieties were coupled to the cross-linker scaffold. ${ }^{12}$ The bulky anionic reactive esters direct acylation to sites that are sterically accessible and cationic. ${ }^{21}$ Reaction of 4.5 eq. of 1 with bovine SOD produces modified SOD with an azide-containing cross-link (SOD- $\mathrm{N}_{3}$ ). The reaction products were analyzed by Sephadex G-200 size-exclusion chromatography (SEC) (Fig. 1). The new peak that appears at approximately $35 \mathrm{~min}$ in the SEC trace is consistent with that expected for cross-linked SOD $\left(\mathrm{SOD}-\mathrm{N}_{3}\right)$. Modification of the lysine residues within the cationic funnel caused the enzyme to elute more quickly by anion exchange chromatography (Fig. 2). The effect of $\mathrm{pH}$ on the yield of $\mathrm{SOD}_{-} \mathrm{N}_{3}$ was examined over the range of $\mathrm{pH}$ 8.0-9.0 in borate buffer solution $(0.01 \mathrm{M})$. Reactions at pH 9.0 over $12 \mathrm{~h}$ gave the most efficient yield of $82 \%$. This is achieved where the amino group is largely present in the unprotonated state, allowing it to react most rapidly with the ester on the cross-linking reagent. SOD-N $\mathrm{N}_{3}$ was then purified by anion

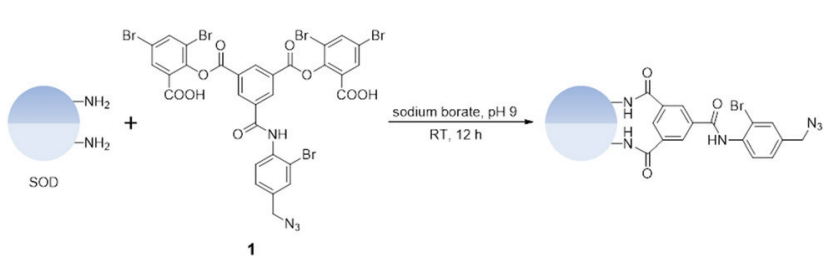

Scheme 1 Site-selective cross-linking of SOD dimer with azide 1 introduces a covalent bridge between each monomer.

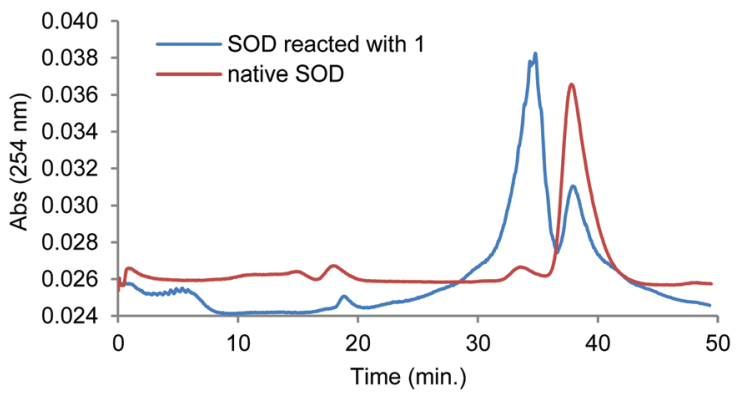

Fig. 1 Sephadex G-200 size-exclusion analysis of SOD under dissociating conditions ( $0.5 \mathrm{M} \mathrm{MgCl}_{2}, 37.5 \mathrm{mM}$ Tris). The peaks at 35 and $39 \mathrm{~min}$ correspond to cross-linked and native SOD, respectively.

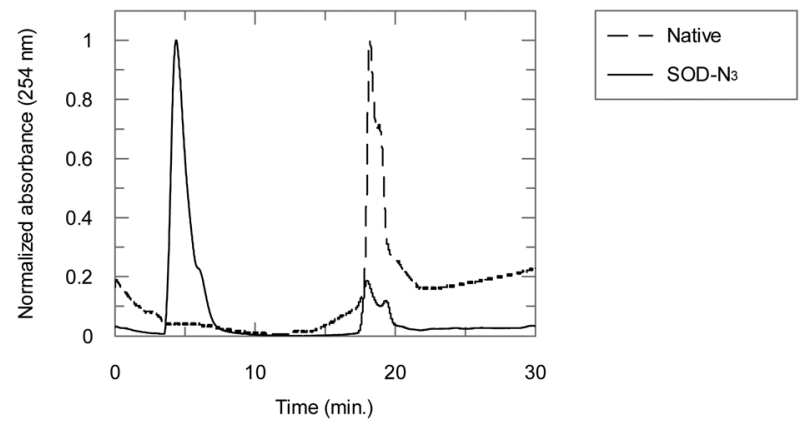

Fig. 2 AX300 anion exchange chromatogram ( $\mathrm{pH}$ 8.0) of SOD before and after modification with cross-linker 1.

exchange chromatography before submission to the PDCuAAC process.

ESI-MS analysis of SOD- $\mathrm{N}_{3}$ provided a mass consistent with a covalently cross-linked SOD dimer with precisely a single cross-linker installed (see Fig. SI $2 \uparrow$ for deconvoluted mass spectrum).

\section{Coupling of cross-linked SOD using PDCuAAC}

Bis-SOD was formed through two sequential $\mathrm{Cu}(\mathrm{I})$ catalysed click reactions (Scheme 2). The $\mathrm{Cu}(\mathrm{I})$ oxidation state is formed

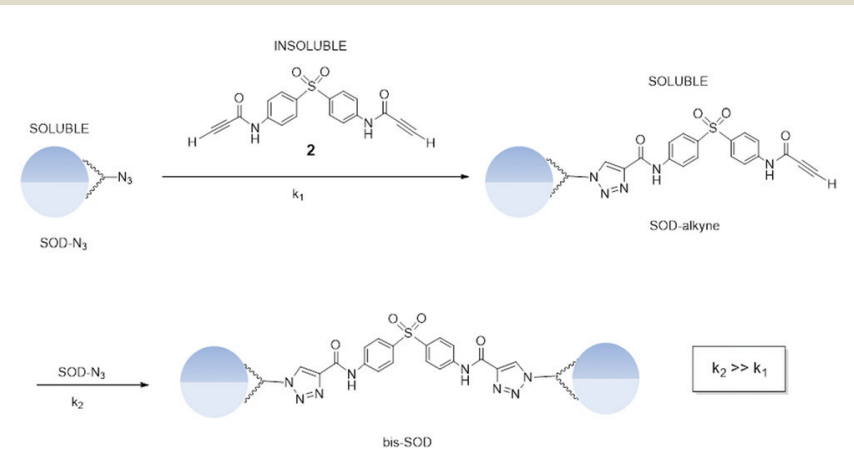

Scheme 2 Reaction of SOD $-\mathrm{N}_{3}$ with initially insoluble bis-alkyne 2 gives the soluble SOD-alkyne, which proceeds to form a bis-SOD. 
in a reducing environment (in the presence of $\mathrm{L}$-ascorbic acid) and maintained by a copper-chelating ligand. To construct the high molecular weight bis-SOD, both ends of the central bisalkyne are required to react. Statistical considerations would normally dictate that a single coupling to the bis-alkyne linker is more likely to occur. However, the use of phase-directed catalysis promotes sequential modification of both ends of the linker over the statistically favoured single modifications. Since any initial CuAAC reaction of a bis-alkyne is limited to the interfacial surface, the first coupling between the bis-alkyne and SOD-N $\mathrm{N}_{3}$ will occur at a relatively slow rate due to the low concentration of the species in reactive proximity. The second coupling reaction of the resulting soluble SOD-alkyne intermediate with SOD- $\mathrm{N}_{3}$ takes place in the same phase, necessarily leading to a greater rate as the accessible relative concentrations are in a common phase. The entire process ensures that both sites of the bis-alkyne linker react to form the bis-tetrazole product (bis-SOD). Thus, the two steps of the coupling process are cooperative.

Ligands accelerate the PDCuAAC reaction and sequester copper ions from other sites of the protein. ${ }^{22}$ However, for our purposes, the catalyst must also be water-soluble due to the mechanism of conjugation. ${ }^{13}$ Several ligands common to bioconjugation were tested for their efficacy in coupling (Fig. 3).

Although TBTA is a commonly used ligand for CuAAC, it is not water-soluble and requires an organic co-solvent. The presence of organic solvent likely increases the solubility of the bis-alkyne linker, sequestering it from the first step of the phase transfer catalysis. Low yields of $\sim 17 \%$ were observed under those conditions.

Sufonated bathophenanthroline (batho) ligand was also tested for use in the coupling reaction. Unlike TBTA, the batho ligand is water soluble. However, a significant drawback is the catalyst's acute oxygen sensitivity. ${ }^{22} \mathrm{Cu}(\mathrm{I})$-batho complexes are highly electron-rich and susceptible to oxidation. The high reactivity of the complex with oxygen can easily consume all of the available reducing agent. ${ }^{23}$ In addition, when working with small volumes $(\sim 50 \mu \mathrm{L})$, creating an oxygen-free environment is difficult. While the reported yield $(\sim 30 \%)$ is an improvement over TBTA, repetitive purging with nitrogen is inefficient.

THPTA is a useful ligand with properties between those of the batho and TBTA ligands. ${ }^{24}$ It is stable under ambient conditions and requires no organic solvent. Subsequent PDCuAAC using THPTA was carried out in $0.02 \mathrm{M}$ phosphate buffer $(\mathrm{pH} 7.4)$ with $\mathrm{SOD}_{3}(0.25 \mathrm{mM})$ in the presence of $2 \mathrm{mM}$ of
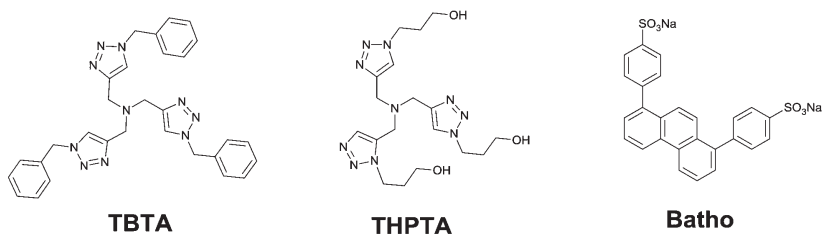

Fig. 3 Structures of the various copper-chelating ligands surveyed for optimization of the formation of bis-SOD by the PDCUAAC.

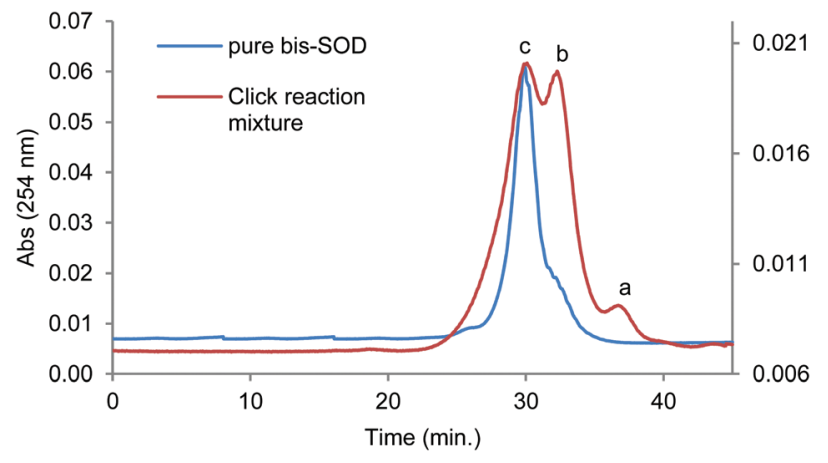

Fig. 4 G200 size-exclusion HPLC analysis of purified and unpurified bis-SOD under dissociating conditions (0.5 $\mathrm{M} \mathrm{MgCl}_{2}, 37.5 \mathrm{mM}$ Tris). Within the reaction mixture contains (a) unmodified SOD, (b) SOD- $\mathrm{N}_{3}$ and (c) bis-SOD.
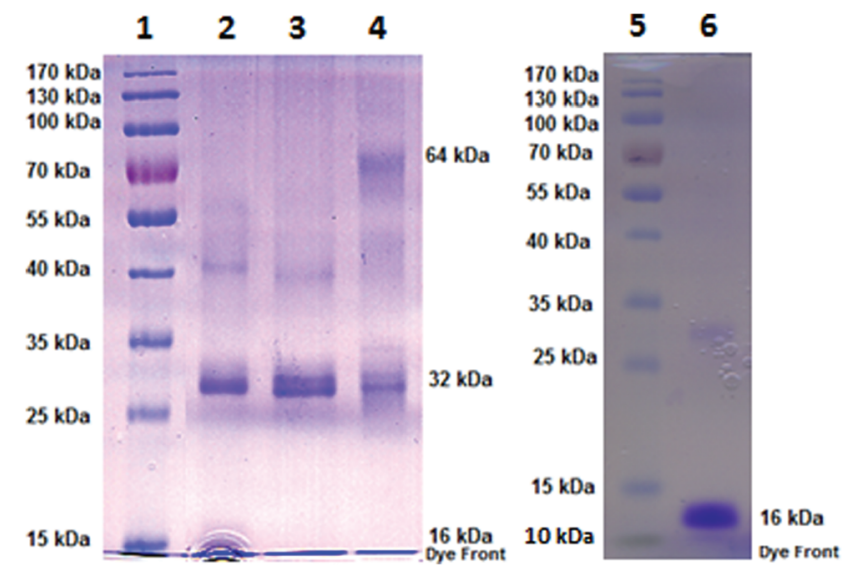

Fig. 5 SDS-PAGE analysis of the various chemically modified SOD species (left) compared to native SOD (right). Lane 1, molecular weight standards; lane 2, SOD- $\mathrm{N}_{3}$ (unpurified); lane 3, SOD- $\mathrm{N}_{3}$ (purified); lane 4, bis-SOD (unpurified); lane 5, molecular weight standards; lane 6, native SOD.

bis-alkyne 2 dissolved in DMSO, $3 \mathrm{mM}$ THPTA ligand, $0.6 \mathrm{mM}$ $\mathrm{CuSO}_{4}$ along with $7 \mathrm{mM}$ ascorbic acid as the reducing agent. Reaction after $4 \mathrm{~h}$ gave a bis-SOD yield of $62 \%$, based on the proportion of bis-SOD relative to the total click reaction mixture (native, modified and bis-SOD), or $72 \%$, based on the proportion of bis-SOD relative to the click reaction participants (SOD- $\mathrm{N}_{3}$ and bis-SOD). The bis-SOD product was observed by size-exclusion HPLC analysis (Fig. 4) and bis-SOD was purified by centrifugal filtration.

\section{SDS-PAGE analysis}

SDS-PAGE analysis of the mixture following the coupling reaction results in a band at $\sim 64 \mathrm{kDa}$ corresponding to the bisSOD product (Fig. 5, lane 4).

\section{Assay of SOD activity}

The activities of SOD- $\mathrm{N}_{3}$ and bis-SOD were measured using the pyrogallol assay (Fig. 6, Table 1). ${ }^{25}$ The differences in activity 


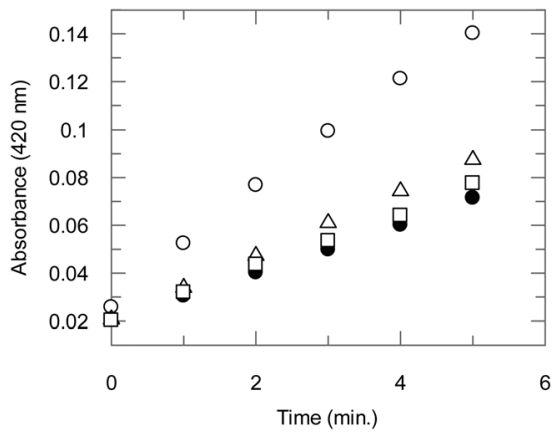

Fig. 6 Rate profile of autoxidation of $4.0 \times 10^{-4} \mathrm{M}$ pyrogallol in the presence of SOD. All samples contain $5.3 \times 10^{-7} \mathrm{~mol} \mathrm{~L}^{-1}$ of the enzyme dimer or 1 unit of SOD.

Table 1 Enzymatic parameters of modified SOD

\begin{tabular}{llrl}
\hline SOD species & \% Inhibition & \% Activity & Specific activity $\left(\mathrm{U} \mathrm{mg}^{-1}\right)$ \\
\hline Native & 50 & 100 & 2078.4 \\
Bis-SOD & 47 & 94 & 1919.3 \\
SOD-N $_{3}$ & 41 & 82 & 1667.2
\end{tabular}

between the modified SODs and native SOD were insignificant, indicating that both the reaction conditions and the introduced chemical modifications do not interfere with the critical catalytic function.

\section{Circular dichroism (CD) analysis of modified SOD}

CD spectra complemented the activity studies, providing evidence that the secondary structure of the protein is maintained after modification. These results together with the activity assay confirm that chemical cross-linking did not distort the cationic funnel surrounding the active site and that coupling did not block the active site of either dimeric constituent (Fig. 7).
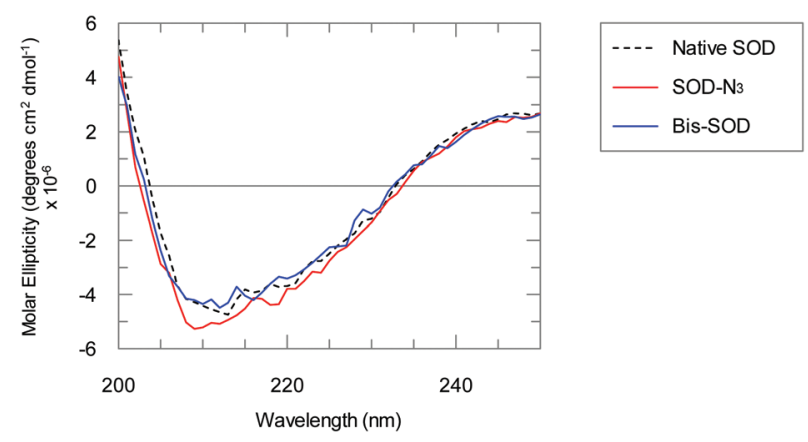

Fig. 7 CD spectrum (200-250 nm) of modified SOD compared to that of native SOD.

\section{Conclusions}

Application of a bioorthogonal strategy that limits initial CuAAC interfacial coupling of cross-linked SOD to a bis alkyne efficiently produces a stable covalent dimer of functional SOD dimers (e.g., a dimer of dimers) as the second CuAAC then occurs rapidly in a common phase. The resulting material is catalytically as competent as the native enzyme with the benefit of a size that would be retained in circulation.

\section{Acknowledgements}

We are grateful for an operating grant from the Canadian Blood Services through the Canadian Institutes of Health Research.

\section{References}

1 R. A. Greenwald, Free Radicals Biol. Med., 1990, 8, 201209.

2 K. Yasui and A. Baba, Inflamm. Res., 2006, 55, 359363.

3 F. D’Agnillo and T. M. S. Chang, Nat. Biotechnol., 1998, 16, 667-671.

4 A. Bayati, O. Kallskog, B. Odlind and M. Wolgast, Acta Physiol. Scand., 1988, 134, 65-74.

5 J. S. Beckman and B. A. Freeman, Biomater. Artif. Cells Artif. Organs, 1988, 15, 761-761.

6 D. V. Ratnam, D. D. Ankola, V. Bhardwaj, D. K. Sahana and M. N. V. R. Kumar, J. Controlled Release, 2006, 113, 189207.

7 M. P. Saifer, R. Somack and L. D. Williams, in Free Radicals in Diagnostic Medicine, ed. D. Armstrong, Springer, US, 1994, ch. 26, vol. 366, pp. 377-387.

8 D. H. Ho, N. S. Brown, A. Yen, R. Holmes, M. Keating, A. Abuchowski, R. A. Newman and I. H. Krakoff, Drug Metab. Dispos., 1986, 14, 349-352.

9 D. D. Lasic, F. J. Martin, A. Gabizon, S. K. Huang and D. Papahadjopoulos, Biochim. Biophys. Acta, 1991, 1070, 187-192.

10 V. V. Rostovtsev, L. G. Green, V. V. Fokin and K. B. Sharpless, Angew. Chem., Int. Ed., 2002, 41, 25962599.

11 A. Alagic, A. Koprianiuk and R. Kluger, J. Am. Chem. Soc., 2005, 127, 8036-8043.

12 A. Wang and R. Kluger, Biochemistry, 2014, 53, 6793-6799.

13 J. S. Foot, F. E. Lui and R. Kluger, Chem. Commun., 2009, 7315-7317.

14 E. M. Sletten and C. R. Bertozzi, Angew. Chem., Int. Ed., 2009, 48, 6974-6998.

15 J. Rappsilber, J. Struct. Biol., 2011, 173, 530-540.

16 M. M. Bradford, Anal. Biochem., 1976, 72, 248-254.

17 U. K. Laemmli, Nature, 1970, 227, 680-685. 
18 S. Marklund and G. Marklund, Eur. J. Biochem., 1974, 47, 469-474.

19 S. J. Kim, D. Han, K. D. Moon and J. S. Rhee, Biosci., Biotechnol., Biochem., 1995, 59, 822-826.

20 L. Magnani, E. M. Gaydou and J. C. Hubaud, Anal. Chim. Acta, 2000, 411, 209-216.

21 R. Kluger and V. De Stefano, J. Org. Chem., 2000, 65, 214219.
22 S. I. Presolski, V. Hong, S.-H. Cho and M. G. Finn, J. Am. Chem. Soc., 2010, 132, 14570-14576.

23 J. E. Hein and V. V. Fokin, Chem. Soc. Rev., 2010, 39, 13021315.

24 V. Hong, S. I. Presolski, C. Ma and M. G. Finn, Angew. Chem., Int. Ed., 2009, 48, 9879-9883.

25 S. Marklund and G. Marklund, Eur. J. Biochem., 1974, 47, 469-474. 